\title{
Some Theorems for Sigma Prime Rings with Differential Identities on Sigma Ideals
}

\author{
Mohd Rais Khan and Mohd Mueenul Hasnain \\ Department of Mathematics, Jamia Millia Islamia, Jamia Nagar, New Delhi 110025, India \\ Correspondence should be addressed to Mohd Mueenul Hasnain; mhamu786@gmail.com
}

Received 26 September 2013; Accepted 28 November 2013

Academic Editors: V. Bovdi, S. Dascalescu, V. Drensky, P. Koshlukov, and S. Yang

Copyright ( 2013 M. R. Khan and M. M. Hasnain. This is an open access article distributed under the Creative Commons Attribution License, which permits unrestricted use, distribution, and reproduction in any medium, provided the original work is properly cited.

\begin{abstract}
There has been considerable interest in the connection between the structure and the $\sigma$-structure of a ring, where $\sigma$ denotes an involution on a ring. In this context, Oukhtite and Salhi (2006) introduced a new class or we can say an extension of prime rings in the form of $\sigma$-prime ring and proved several well-known theorems of prime rings for $\sigma$-prime rings. A continuous approach in the direction of $\sigma$-prime rings is still on. In this paper, we establish some results for $\sigma$-prime rings satisfying certain identities involving generalized derivations on $\sigma$-ideals. Finally, we give an example showing that the restrictions imposed on the hypothesis of the various theorems were not superfluous.
\end{abstract}

\section{Introduction}

Throughout the paper, $R$ will denote an associative ring with center $Z(R)$. For any $x, y \in R$, the symbol $[x, y]$ stands for the Lie product $x y-y x$ and the symbol $x \circ y$ denotes the Jordan product $x y+y x$. A ring $R$ is called 2-torsion free, if whenever $2 x=0$, with $x \in R$, then $x=0$. Recall that a ring $R$ is prime if, for any $x, y \in R, x R y=0$ implies $x=0$ or $y=0$. A ring $R$ equipped with an involution $\sigma$ is to be $\sigma$ prime if $x R y=x R \sigma(y)=0 \Rightarrow x=0$ or $y=0$. An example, according to Oukhtite and Salhi [1], shows that every prime ring can be injected in $\sigma$-prime ring and from this point of view $\sigma$-prime rings constitute a more general class of prime rings. An ideal $U$ is a $\sigma$-ideal if $U$ is invariant under $\sigma$; that is, $\sigma(U)=U$. Note that an ideal may not be a $\sigma$-ideal. Let $S$ be a ring of integers and $R=S \times S$. Consider a map $\sigma: R \rightarrow R$ defined by $\sigma((x, y))=(y, x)$ for all $x, y \in R$. For an ideal $A=S \times\{0\}$ of $R, A$ is not a $\sigma$-ideal of $R$ since $\sigma(A)=\{0\} \times S \neq A$.

Several authors have studied the relationship between the commutativity of a ring and the behavior of a special mapping on that ring. In particular, there has been considerable interest in centralizing automorphisms and derivations defined on rings (see, e.g., [2-4], where further references can be found).
As defined in [5, 6], an additive mapping $F: R \rightarrow R$ is called generalized derivation with associated derivation $D$ if

$$
F(x y)=F(x) y+x D(y)=D(x) y+x F(y), \quad \forall x, y \in R .
$$

Familiar examples of generalized derivations are derivations and generalized inner derivations and later included left multiplier, that is, an additive mapping $F: R \rightarrow R$ satisfying $F(x y)=F(x) y$ for all $x, y \in R$. Since the sum of two generalized derivations is a generalized derivation, every map of the form $F(x)=c x+D(x)$, where $c$ is a fixed element of $R$ and $D$, a derivation of $R$, is a generalized derivation, and if $R$ has 1, all generalized derivations have this form.

In 2006, Oukhtite and Salhi [1] introduced a new class or we can say an extension of prime rings in the form of $\sigma$-prime ring. However, the actual motivation behind their first successful work came from Posner's [7] second theorem only. In [8], they successfully extended the result for $\sigma$-prime ring. Recently, a major breakthrough has been achieved by Oukhtite and Salhi [9], where the important results by Posner, Herstein, and Bell have been proved for $\sigma$-prime rings. More precisely, Posner's second theorem of existence of a nonzero centralizing derivation on prime ring which makes the ring 
commutative if $R$ is a prime ring of characteristic $\neq 2$ with a nonzero derivation $D$ such that $[D(x), D(y)]=0$ for all $x, y \in R$. Oukhtite and Salhi [9, Theorem 1.2] proved that the same result holds for $\sigma$-prime rings. Motivated by a well-known result by Herstein [10], Bell and Daif [11] studied derivation $D$ satisfying $D(x y)=D(y x)$ for all $x, y \in R$. This result has been extended for $\sigma$-prime rings [9, Theorem 1.3]. They initiated their work from [1], continued in $[12,13]$, and are spree of developing and extending more and more results which hold true for a prime ring. A continuous approach in this direction is still on. In this paper, we continue the study in the direction of Oukhtite by providing some results which are of independent interest and related to generalized derivations for $\sigma$-prime rings. More precisely, we will prove the following results.

Theorem 1. Suppose that $R$ is a 2-torsion free $\sigma$-prime ring and $U \neq 0$ a $\sigma$-ideal of $R$. If $R$ admits a generalized derivation $(F, D)$ with the additional condition that $\sigma D=D \sigma$ such that $D(x)$ 。 $F(y)=[x, y]$ for all $x, y \in U$ or $D(x) \circ F(y)=-[x, y]$ for all $x, y \in U$, then $F$ is a left multiplier.

Theorem 2. Suppose that $R$ is a 2-torsion free $\sigma$-prime ring and $U \neq\{0\}$ a $\sigma$-ideal of $R$. If $R$ admits a generalized derivation $(F, D)$ with the additional condition that $\sigma D=D \sigma$ such that $F[x, y]=F(x) \circ y+D(y) \circ x$ for all $x, y \in U$, then $D=F=0$.

Theorem 3. Suppose that $R$ is a 2-torsion free $\sigma$-prime ring and $U \neq\{0\}$ a $\sigma$-ideal of $R$. If $R$ admits a generalized derivation $(F, D)$ with the additional condition that $\sigma D=D \sigma$ such that $F(x \circ y)=[F(x), y]+[D(y), x]$ for all $x, y \in U$, then $D=F=$ 0 .

Theorem 4. Suppose that $R$ is a 2-torsion free $\sigma$-prime ring and $U \neq\{0\}$ a $\sigma$-ideal of $R$. If $R$ admits a generalized derivation $(F, D)$ with the additional condition that $\sigma D=D \sigma$ such that $F(x \circ y)=[F(x), y]$ or $F(x \circ y)=-[F(x), y]$ for all $x, y \in U$, then $D=F=0$.

Theorem 5. Suppose that $R$ is a 2-torsion free $\sigma$-prime ring and $U \neq\{0\}$ a $\sigma$-ideal of $R$. If $R$ admits a generalized derivation $(F, D)$ with the additional condition that $\sigma D=D \sigma$ such that $F[x, y]=F(x) \circ y$ or $F[x, y]=-F(x) \circ y$ for all $x, y \in U$, then $D=F=0$.

We will make extensive use of the following basic identities without any specific mention; for all $x, y, z \in R$,

(i) $[x y, z]=x[y, z]+[x, z] y \&[x, y z]=y[x, z]+$ $[x, y] z$,

(ii) $x \circ(y z)=(x \circ y) z-y[x, z]=y(x \circ z)+[x, y] z$,

(iii) $(x y) \circ z=(x \circ z) y+x[y, z]=x(y \circ z)-[x, z] y$.

\section{Proof of Main Results}

In all that follows, $S a_{\sigma}(R)$ will denote the set of symmetric and skew-symmetric elements of $R$; that is, $S a_{\sigma}(R)=\{x \in R \mid$ $\sigma(x)= \pm x\}$. We begin with the following results which will be used later to prove our theorems.
Lemma 6 (see [14, Lemma 3.1]). Let $R$ be a 2-torsion free $\sigma$ prime ring and let $U \neq 0$ be a $\sigma$-ideal of $R$. If $x, y \in R$ such that $x U y=x U \sigma(y)=\{0\}$, then $x=0$ or $y=0$.

Lemma 7 (see [9, Lemma 2.3]). Let $R$ be a 2-torsion free $\sigma$ prime ring and let $U \neq 0$ be a $\sigma$-ideal of $R$. If $D$ is a derivation on $R$ with the additional condition that $\sigma D=D \sigma$ such that $D^{2}(U)=0$, then $D=0$.

Lemma 8 (see [14, Theorem 3.2]). Let $R$ be a 2-torsion free $\sigma$ prime ring and let $U \neq 0$ be a $\sigma$-ideal of $R$. If $D$ is a derivation on $R$ with the additional condition that $\sigma D=D \sigma$ such that $D(U) \subset Z(R)$, then $R$ is commutative.

Proof of Theorem 1. From hypothesis, we have

$$
D(x) \circ F(y)=[x, y] \quad \forall x, y \in U .
$$

Replacing $y$ with $y r$ where $r \in R$ in (2) and using it, we get

$$
\begin{aligned}
& (D(x) \circ y) D(r)-y[D(x), D(r)] \\
& \quad-F(y)[D(x), r]-y[x, r]=0 \quad \forall x, y \in U .
\end{aligned}
$$

Substituting $z y$ for $y$ in (3) and using (3), we arrive at

$$
\begin{array}{r}
{[D(x), z] y D(r)-D(z) y[D(x), r]=0} \\
\forall x, y, z \in U r \in R .
\end{array}
$$

Putting $r=D(x)$ in (4), we obtain

$$
[D(x), z] y D^{2}(x)=0 \quad \forall x, y, z \in U .
$$

This implies that

$$
[D(x), z] U D^{2}(x)=0 \quad \forall x, z \in U .
$$

For all $x \in U \cap S_{a_{\sigma}}(R)$, as $D$ commutes with $\sigma$ then (6) is forced to $[D(x), z] U \sigma\left(D^{2}(x)\right)=\{0\}$ and Lemma 6 yields $D^{2}(x)=0$ or $[D(x), U]=\{0\}$. Let $x \in U$; since $x-\sigma(x) \in U \cap S_{a_{\sigma}}(R)$, then we have $D^{2}(x-\sigma(x))=0$ or $[D(x-\sigma(x)), U]=\{0\}$. If $[D(x-\sigma(x)), U]=0$, then (6) implies

$$
\sigma([D(x), z]) U D^{2}(x)=0 \quad \forall z \in U .
$$

Equations (7) and (6), by Lemma 6, imply $D^{2}(x)=0$ or $[D(x), U]=\{0\}$. Similarly, if $D^{2}(x-\sigma(x))=0$, then $D^{2}(x)=$ $\sigma\left(D^{2}(x)\right)$ and using (6) we have $[D(x), z] U \sigma\left(D^{2}(x)\right)=0$ for all $z \in U$. Once again using Lemma 6 , the last equality together with (6) leads to $D^{2}(x)=0$ or $[D(x), U]=\{0\}$. Hence, in both cases we have $D^{2}(x)=0$ or $[D(x), U]=\{0\}$ for all $x \in U$. Since a group cannot be the union of proper subgroups, according to Brauer's trick, either $D^{2}(x)=0$ or $[D(x), U]=\{0\}$. Taking $D^{2}(x) \neq 0$ together with Lemma 7 , we are forced to consider that

$$
[D(x), U]=\{0\} \quad \forall x \in U .
$$

Then, we conclude, by Lemma 8 , that $R$ is commutative. Hence, our hypothesis becomes

$$
2 D(x) F(y)=0 \quad \forall x, y \in U .
$$


Using 2-torsion freeness, we have

$$
D(x) F(y)=0, \quad \forall x, y \in U,
$$

so that

$$
D(x) R F(y)=\sigma(D(x)) R F(y)=\{0\}, \quad \forall x, y \in U .
$$

Since $R$ is prime and $D \neq 0$, (11) yields $F(y)=0$ for all $y \in$ $U$ and so $F=0$ by simple calculation. From $0=F(x n)=$ $F(x) n+x D(n)$, it then follows that $x D(n)=0$ for all $x \in U, n \in$ $N$, and therefore $D=0$ by Lemma 6 , which is a contradiction. Consequently, we take $D^{2}(x)=0$ and so $D=0$ by Lemma 7 . Hence, this completes the proof.

Similar arguments can be adapted in the case $D(x)$ 。 $F(y)=-[x, y]$ and we can omit the similar proof.

Proof of Theorem 2. From hypothesis, we have

$$
F[x, y]=F(x) \circ y+D(y) \circ x \quad \forall x, y \in U .
$$

Taking $y x$ instead of $y$ in (12) and using it, we obtain

$$
2[x, y] D(x)=-y[F(x), x]-y(D(x) \circ x) \quad \forall x, y \in U .
$$

Replacing $y$ with $y z$ in (13) and in view of (13), we get

$$
2[x, y] z D(x)=0 \quad \forall x, y, z \in U .
$$

Using 2-torsion freeness, we have

$$
[x, y] z D(x)=0 \quad \forall x, y, z \in U,
$$

so that

$$
[x, y] U D(x)=\{0\} \quad \forall x, y \in U .
$$

For all $x \in U \cap S a_{\sigma}(R)$, as $D$ commutes with $\sigma$, then (16) forces $[x, y] U \sigma D(x)=\{0\}$ and from Lemma 6, the last equality yields $[x, y]=0$ for all $y \in U$ or $D(x)=0$. Since a group cannot be the union of proper subgroups, according to Brauer's trick either $[x, y]=0$ for all $y \in U$ or $D(x)=0$. Supposing that $D(x) \neq 0$ and by using similar reasoning to the one in [1, Proof of Theorem 1.1] yield that $R$ is commutative. Hence, using 2-torsion freeness, our hypothesis becomes $0=$ $F(x) y+x D(y)$; that is, $F(x y)=0$ for all $x, y \in U$. From $0=F(x y n)=F((x y) n)=F(x y) n+x y D(n)$, it follows that $x y D(n)=0$ for all $x, y \in U, n \in N$. Therefore,

$$
x U D(n)=\sigma(x) U D(n)=\{0\} .
$$

It follows from Lemma 6 that $D=0$, which is a contradiction. Consequently, we take $D=0$ and $F$ acts as a left multiplier. In this situation, our hypothesis $F[x, y]=F(x) \circ y$ is forced to

$$
F(y x)=-y F(x) \quad \forall x, y \in U
$$

Clearly, $F(x y z)=F((x y) z)=-x y F(z)$ and also we obtain $F(x y z)=F(x(y z))=-x F(y z)=x y F(z)$, comparing these two expressions for $F(x y z)$ gives us $x y F(z)=0$ for all $x, y, z \in U$ by 2-torsion freeness. That is, $x U F(z)=\{0\}$ for all $x, z \in U$ which leads to $F=0$ by Lemma 6 . This completes the proof.
Proof of Theorem 3. From hypothesis, we have

$$
F(x \circ y)=[F(x), y]+[D(y), x] \quad \forall x, y \in U .
$$

Taking $y x$ instead of $y$ in (19) and using it, we obtain

$$
(x \circ y) D(x)=y[F(x), x]+y[D(x), x]+[y, x] D(x)
$$

$$
\forall x, y \in U \text {. }
$$

Replacing $y$ with $y z$ in (20) and using it, we have

$$
2[x, y] z D(x)=0 \quad \forall x, y, z \in U .
$$

Since $R$ is 2 -torsion free, we find

$$
[x, y] z D(x)=0 \quad \forall x, y, z \in U,
$$

so that

$$
[x, y] U D(x)=\{0\} \quad \forall x, y \in U,
$$

The last equality is the same as (16) in the proof of Theorem 2. Thus, by using the same arguments as in the proof of Theorem 2 , we can conclude the result here.

Proof of Theorem 4. From hypothesis, we have

$$
F(x \circ y)=[F(x), y] \quad \forall x, y \in U .
$$

Replacing $y$ by $y x$ in (24) and using it, we find

$$
(x \circ y) D(x)=y[F(x), x] \quad \forall x, y \in U .
$$

Taking $y z$ instead of $y$ in (25) and using it, we get

$$
[x, y] z D(x)=0 \quad \forall x, y, z \in U,
$$

so that

$$
[x, y] U D(x)=\{0\} \quad \forall x, y \in U .
$$

Equation (27) is the same as (16) in the proof of Theorem 2. Thus, by using the same arguments as in the proof of Theorem 2 , we can conclude the result here.

Similar arguments can be adapted in the case $F(x \circ y)=$ $-[F(x), y]$ and we can omit the same proof.

Proof of Theorem 5. From hypothesis, we have

$$
F[x, y]=F(x) \circ y \quad \forall x, y \in U .
$$

Replacing $y$ with $y x$ in (28) and using it, we find

$$
[x, y] D(x)=-y[F(x), x] \quad \forall x, y \in U .
$$

Putting $y=y z$ in (29) and using it, we get

$$
[x, y] z D(x)=0 \quad \forall x, y, z \in U,
$$

so that

$$
[x, y] U D(x)=\{0\} \quad \forall x, y \in U .
$$

The last equality is the same as (27) same as (16) in the proof of Theorem 4. Thus, by using the same arguments as in the proof of Theorem 4, we can conclude the result here.

Application of similar arguments to the one mentioned above yields the case $F[x, y]=-F(x) \circ y$ and we can omit the similar proof. 


\section{Homomorphism or Antihomomorphism}

Suppose $\alpha$ and $\beta$ are two endomorphisms of $R$. An additive mapping $f: R \rightarrow R$ is called generalized $(\alpha, \beta)$-derivation associated with $(\alpha, \beta)$-derivation $d$, where $d$ is defined as $d(x y)=\beta(x) d(y)+d(x) \alpha(y)$ for all $x, y \in R$ if $f(x y)=$ $f(x) \alpha(y)+\beta(x) d(y)=d(x) \alpha(y)+\beta(x) f(y)$ for all $x, y \in R$. Obviously, every $(I, I)$-derivation on $R$ is just a generalized derivation on $R$, where $I$ is the identity mapping. If $(f, d)$ is a generalized $(\alpha, \beta)$-derivation of $R$ and $f(a b)=f(a) f(b)$ or $f(a b)=f(b) f(a)$ for all $a, b \in A$, then $f$ is called a generalized $(\alpha, \beta)$-derivation which acts as a homomorphism or antihomomorphism on $A$, where $A$ is a nonempty subset of $R$.

Recently, Bell and Kappe [15] proved that if $\delta$ is a derivation of prime ring $\Re$ which acts as a homomorphism or an antihomomorphism on a nonzero ideal $I$ of $\mathfrak{R}$, then $\delta=0$ on $\mathfrak{R}$. Thereafter, Albaş and Argaç [2] extended this result to generalized derivation. Further, Oukhtite and Salhi [1] proved that the above result is also true for $\sigma$-prime rings. It is natural to raise a question: is the above result valid in generalized $(\alpha, \beta)$-derivation? In this context, we give an affirmative answer to the question.

Theorem 9. Suppose that $R$ is a 2-torsion free $\sigma$-prime ring, $U \neq 0$ is a $\sigma$-ideal, and $(f \neq 0, d)$ is a generalized $(\alpha, \beta)$ derivation with the additional condition that $\sigma D=D \sigma$, where $\beta$ is an automorphism of $R$ such that $\sigma \beta=\beta \sigma$. If $f$ acts as a homomorphism or as an antihomomorphism on $U$, then $d=0$ or $f=\beta$.

Proof

Step I. Let $f$ act as a homomorphism on $U$; then we have

$$
\begin{array}{r}
f(x) f(y)=f(x y)=f(x) \alpha(y)+\beta(x) d(y) \\
\forall x, y \in U .
\end{array}
$$

Replacing $x$ with $x z$ in (32), we get

$$
\begin{array}{r}
f(x z) f(y)=f(x z) \alpha(y)+\beta(x z) d(y) \\
=f(x) f(z) \alpha(y)+\beta(x z) d(y) \\
\forall x, y, z \in U,
\end{array}
$$

so that

$$
\begin{aligned}
f(x z) & f(y) \\
= & f(x) f(z) \alpha(y)+\beta(x z) d(y) \quad \forall x, y, z \in U .
\end{aligned}
$$

Since $f$ is a homomorphism on $U$, we have

$$
\begin{aligned}
& f(x z) f(y)=f(x) f(z y) \\
&=f(x) f(z) \alpha(y)+f(x) \beta(z) d(y) \\
& \forall x, y, z \in U .
\end{aligned}
$$

From (34) and (35), we have

$$
(f(x)-\beta(x)) \beta(z) d(y)=0, \quad \forall x, y, z \in U,
$$

and hence $(f(x)-\beta(x)) \beta(U) d(y)=\{0\}$. Letting $V=\beta(U)$, it is easy to see that $V$ is a nonzero $\sigma$-ideal; that is,

$$
(f(x)-\beta(x)) V d(y)=\{0\} \quad \forall x, y \in U .
$$

Now (37) yields $(f(x)-\beta(x)) V d(y)=(f(x)-$ $\beta(x)) V \sigma(d(y))=\{0\}$. As $d$ commutes with $\sigma$ and $V$ is a $\sigma$-ideal, then, by Lemma 6 , we have either $f(x)-$ $\beta(x)=0$ or $d(y)=0$ for all $x, y \in U$; namely, $f=\beta$ or $d=0$ on $U$.

Step II. Letting $f$ act as an antihomomorphism on $U$, then we have

$$
\begin{array}{r}
f(y) f(x)=f(x y)=f(x) \alpha(y)+\beta(x) d(y) \\
\forall x, y \in U .
\end{array}
$$

Replacing $x$ by $x y$ in (38), we find

$$
\begin{aligned}
f(y) & f(x) \alpha(y)+f(y) \beta(x) d(y) \\
& =f(y) f(x) \alpha(y)+\beta(x y) d(y) \quad \forall x, y \in U,
\end{aligned}
$$

so that

$$
f(y) \beta(x) d(y)=\beta(x) \beta(y) d(y) \quad \forall x, y \in U .
$$

Putting $x=z x$ in (40) and using it, we arrive at

$$
[f(y), \beta(z)] \beta(x) d(y)=0 \quad \forall x, y \in U .
$$

As $\beta$ is an automorphism, that is, if we set $V=\beta(U)$, (41) yields

$$
[f(y), \beta(z)] V d(y)=\{0\} \quad \forall x, y \in U .
$$

For all $y \in U \bigcap S a_{\sigma}(R)$ and from (42), we have

$$
\begin{aligned}
{[f(y), \beta(z)] V d(y)=[f(y), \beta(z)] V \sigma(d(y)) } & =\{0\} \\
\forall z & \in U .
\end{aligned}
$$

In view of Lemma 6 , the last equality yields $f(y) \in$ $Z(R)$ or $d(y)=0$. Now, assume that $d(x)=0$. Then $f$ is a left multiplier and (38) yields

$$
f(y) f(x)=f(x) \alpha(y) \quad \forall x \in U .
$$

Taking $y x$ instead of $y$ in (44) and using it, we reach the following:

$$
(f(y) \alpha(x)-f(x) f(y)) f(x)=0 \quad \forall x \in U .
$$

As $U$ is a $\sigma$-ideal and from Lemma 6 , we get

$$
f(x) f(y)=f(y) \alpha(x) \quad \forall x \in U .
$$

Comparing (44) and (46), we have

$$
f(y) f(x)=f(x) f(y) \quad \forall x \in U .
$$

Therefore, in both cases we find $f(y) f(x)=f(x) f(y)$ for all $x \in U$. Thus, $f$ acts as a homomorphism and hence, by the previous part of this theorem, we conclude that $f=\beta d=$ 0 . 


\section{Counter Example}

Here, we try to construct some examples to demonstrate that the above results are not true in the case of arbitrary ring.

Example 1. Let

$$
R=\left\{\left(\begin{array}{ll}
a & b \\
0 & c
\end{array}\right): a, b, c \in A\right\},
$$

where $A$ is a ring of integer and

$$
U=\left\{\left(\begin{array}{ll}
0 & b \\
0 & 0
\end{array}\right): b \in A\right\}
$$

We define the following maps:

$$
\begin{gathered}
\sigma\left(\begin{array}{ll}
a & b \\
0 & c
\end{array}\right)=\left(\begin{array}{cc}
c & -b \\
0 & a
\end{array}\right), \quad F\left(\begin{array}{ll}
a & b \\
0 & c
\end{array}\right)=\left(\begin{array}{cc}
a & 2 b \\
0 & 0
\end{array}\right), \\
D\left(\begin{array}{ll}
a & b \\
0 & c
\end{array}\right)=\left(\begin{array}{ll}
0 & b \\
0 & 0
\end{array}\right) .
\end{gathered}
$$

Then it can be seen easily that $U$ is a $\sigma$-ideal of $R$ with involution $\sigma$ and $(F, D)$ is a generalized derivation with additional condition that $\sigma D=D \sigma$. Moreover, it is straightforward to check that $F$ satisfies the following properties: (i) $D(x)$ 。 $F(y)= \pm[x, y]$, (ii) $F[x, y]=F(x) \circ y+D(y) \circ x$, (iii) $F(x \circ y)=[F(x), y]+[D(y), x]$, (iv) $F(x \circ y)= \pm[F(x), y]$, (v) $F[x, y]= \pm(F(x) \circ y)$ for all $x, y \in U$. However, neither $D=F=0$ nor $F$ is a left multiplier.

Example 2. Let

$$
R=\left\{\left(\begin{array}{ll}
a & 0 \\
b & c
\end{array}\right): a, b, c \in A\right\}
$$

where $A$ is a ring of integer and

$$
U=\left\{\left(\begin{array}{ll}
0 & 0 \\
b & 0
\end{array}\right): b \in A\right\} .
$$

We define the following maps:

$$
\begin{gathered}
\sigma\left(\begin{array}{ll}
a & 0 \\
b & c
\end{array}\right)=\left(\begin{array}{cc}
c & 0 \\
-b & a
\end{array}\right), \quad F\left(\begin{array}{ll}
a & 0 \\
b & c
\end{array}\right)=\left(\begin{array}{ll}
a & 0 \\
0 & c
\end{array}\right), \\
\alpha\left(\begin{array}{ll}
a & 0 \\
b & c
\end{array}\right)=\left(\begin{array}{ll}
0 & 0 \\
0 & c
\end{array}\right), \quad \beta\left(\begin{array}{ll}
a & 0 \\
b & c
\end{array}\right)=\left(\begin{array}{ll}
a & 0 \\
0 & 0
\end{array}\right), \\
D\left(\begin{array}{ll}
a & b \\
0 & c
\end{array}\right)=\left(\begin{array}{ll}
a & 0 \\
0 & 0
\end{array}\right) .
\end{gathered}
$$

Then it can be seen easily that $U$ is a $\sigma$-ideal of $R$ with involution $\sigma$ and $(F, D)$ is a generalized derivation with the additional condition that $\sigma D=D \sigma$. Moreover, it is straightforward to check that $F$ acts as a homomorphism and antihomomorphism on $U$ and also we observe that $\sigma \beta=\beta \sigma$. However, neither $D=0$ nor $F=\beta$.

\section{References}

[1] L. Oukhtite and S. Salhi, "On generalized derivations of 6-prime rings," African Diaspora Journal of Mathematics, vol. 5, pp. 1923, 2006.

[2] E. Albaş and N. Argaç, "Generalized derivations of prime rings," Algebra Colloquium, vol. 11, no. 3, pp. 399-410, 2004.

[3] M. Ashraf and S. Ali, "On generalized Jordan left derivations in rings," Bulletin of the Korean Mathematical Society, vol. 45, no. 2, pp. 253-261, 2008.

[4] M. Ashraf, A. Ali, and R. Rani, "On generalized derivations of prime rings," Southeast Asian Bulletin of Mathematics, vol. 29, no. 4, pp. 669-675, 2005.

[5] M. Brešar, "On the distance of the composition of two derivations to the generalized derivations," Glasgow Mathematical Journal, vol. 33, no. 1, pp. 89-93, 1991.

[6] B. Hvala, "Generalized derivations in rings," Communications in Algebra, vol. 26, no. 4, pp. 1147-1166, 1998.

[7] E. C. Posner, "Derivations in prime rings," Proceedings of the American Mathematical Society, vol. 8, pp. 1093-1100, 1957.

[8] L. Oukhtite and S. Salhi, "Derivations and commutativity of $\sigma$ prime rings," International Journal of Contemporary Mathematical Sciences, vol. 1, no. 9-12, pp. 439-448, 2006.

[9] L. Oukhtite and S. Salhi, "On derivations in $\sigma$-prime rings," International Journal of Algebra, vol. 1, no. 5-8, pp. 241-246, 2007.

[10] I. N. Herstein, Rings with Involution, Chicago Lectures in Mathematics, The University of Chicago Press, Chicago, Ill, USA, 1976.

[11] H. E. Bell and M. N. Daif, "On derivations and commutativity in prime rings," Acta Mathematica Hungarica, vol. 66, no. 4, pp. 337-343, 1995.

[12] L. Oukhtite, "An extension of Posner's second theorem to rings with involution," International Journal of Modern Mathematics, vol. 4, no. 3, pp. 303-308, 2009.

[13] L. Oukhtite, S. Salhi, and L. Taoufiq, "Generalized derivations and commutativity of rings with involution," Beiträge zur Algebra und Geometrie, vol. 51, no. 2, pp. 345-351, 2010.

[14] L. Oukhtite and S. Salhi, "On commutativity of $\sigma$-prime rings," Glasnik Matematički, vol. 41, no. 1, pp. 57-64, 2006.

[15] H. E. Bell and L.-C. Kappe, "Rings in which derivations satisfy certain algebraic conditions," Acta Mathematica Hungarica, vol. 53, no. 3-4, pp. 339-346, 1989. 


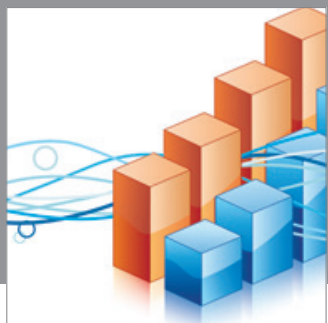

Advances in

Operations Research

mansans

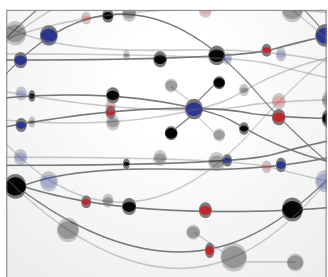

The Scientific World Journal
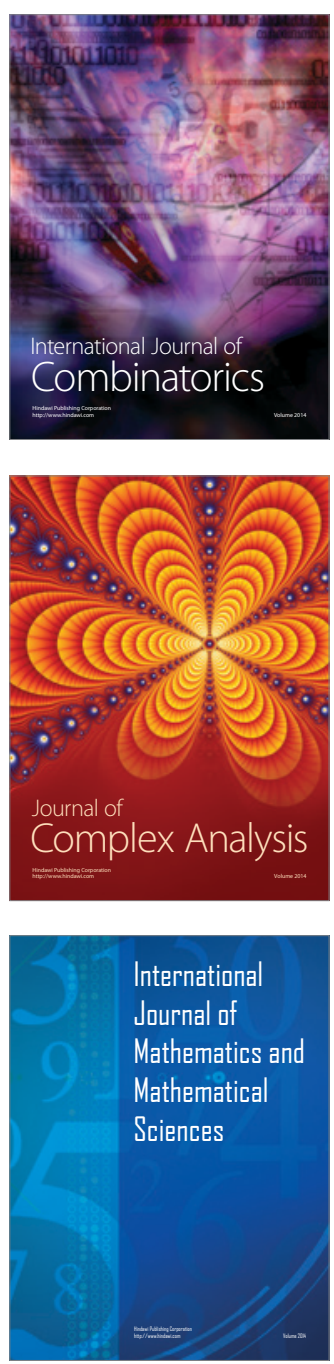
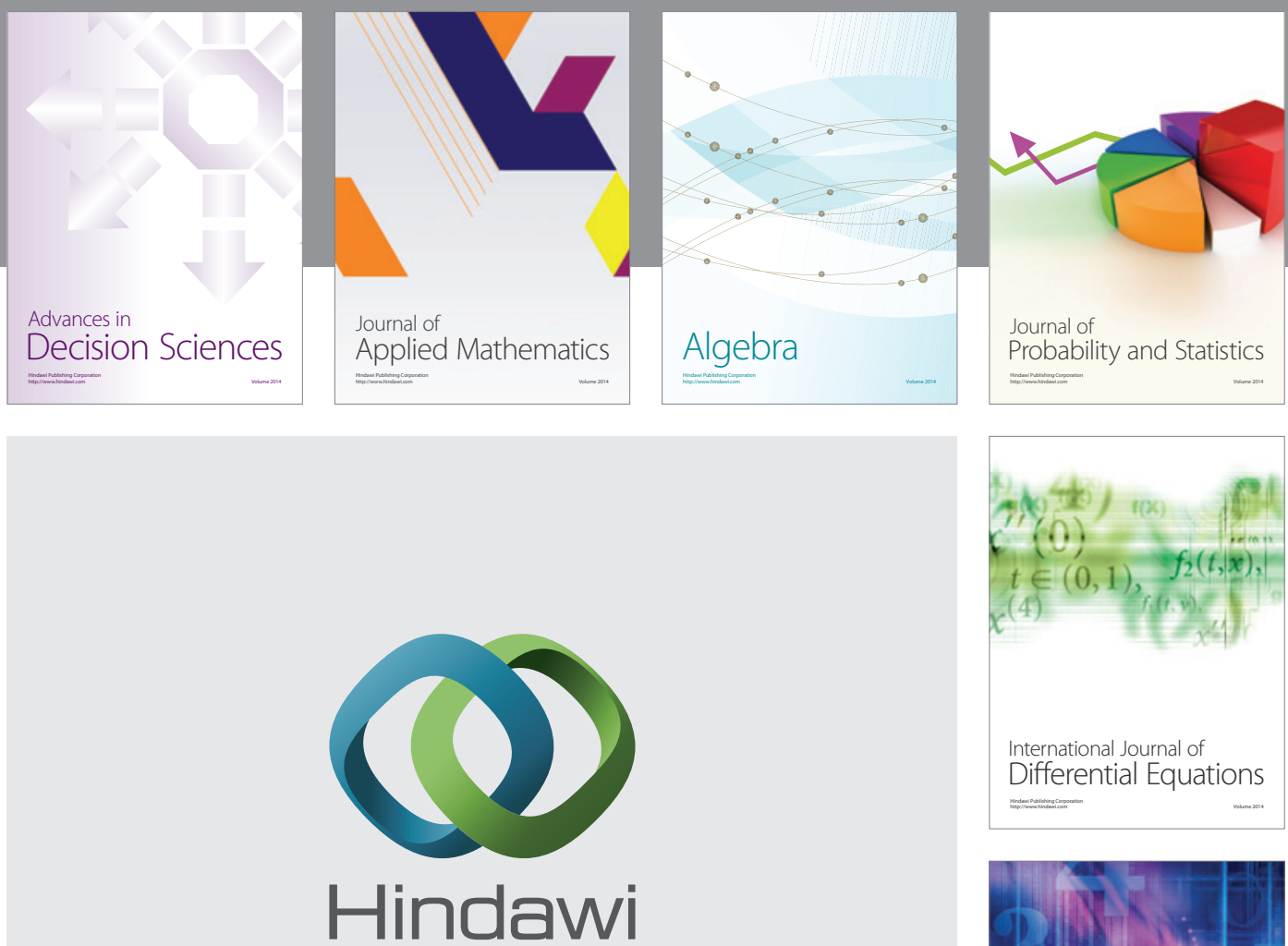

Submit your manuscripts at http://www.hindawi.com
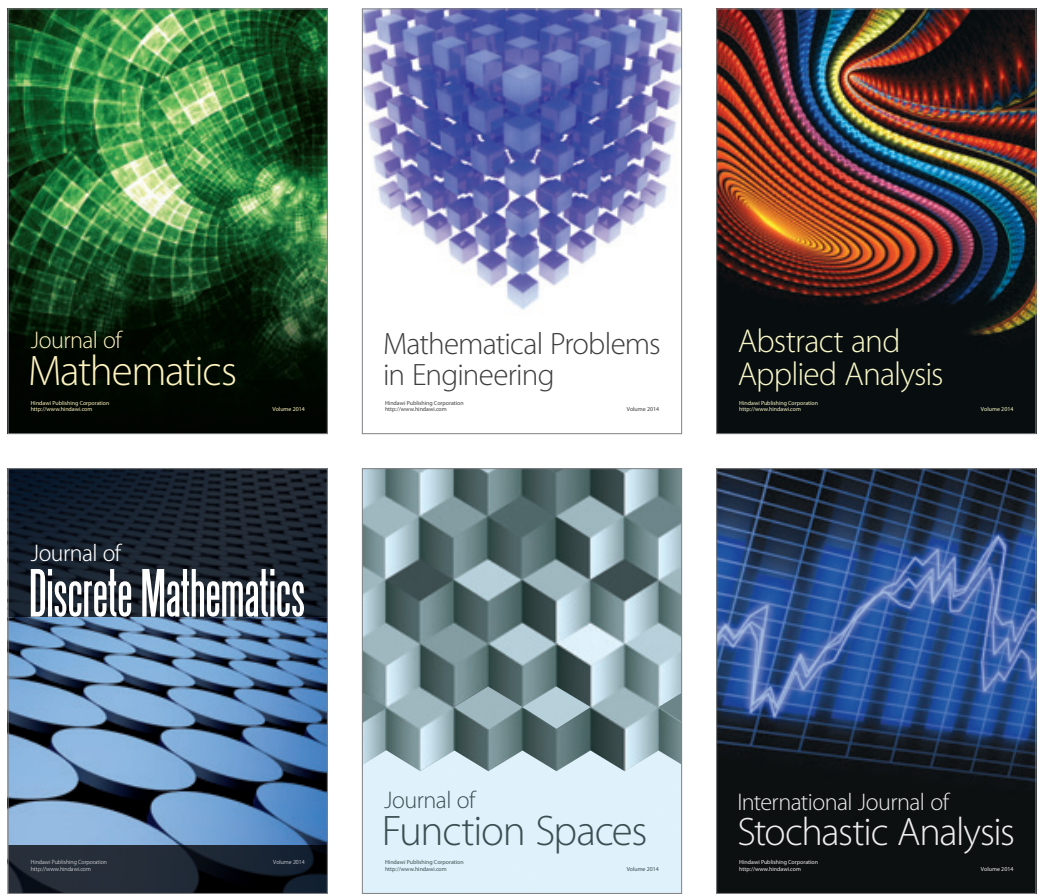

Journal of

Function Spaces

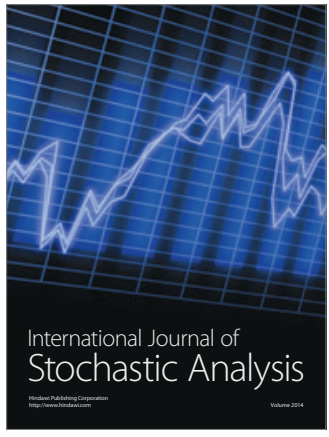

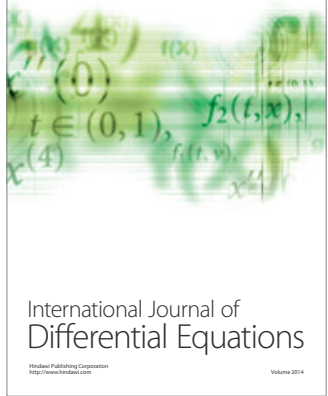
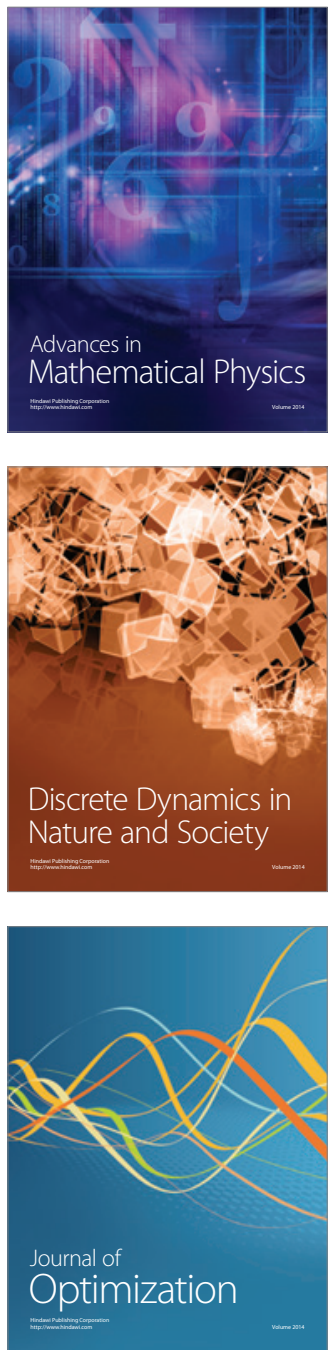